\title{
Optimal Pricing of Spectrum Resources in Wireless Opportunistic Access
}

\author{
Hanna Bogucka \\ Chair of Wireless Communications, Poznan University of Technology, ul. Polanka 3, 60-965 Poznan, Poland \\ Correspondence should be addressed to Hanna Bogucka, hbogucka@et.put.poznan.pl
}

Received 8 February 2012; Accepted 23 April 2012

Academic Editor: Luca Ronga

Copyright ( 2012 Hanna Bogucka. This is an open access article distributed under the Creative Commons Attribution License, which permits unrestricted use, distribution, and reproduction in any medium, provided the original work is properly cited.

\begin{abstract}
We consider opportunistic access to spectrum resources in cognitive wireless networks. The users equipment, or the network nodes in general are able to sense the spectrum and adopt a subset of available resources (the spectrum and the power) individually and independently in a distributed manner, that is, based on their local channel quality information and not knowing the Channel State Information (CSI) of the other nodes' links in the considered network area. In such a network scenery, the competition of nodes for available resources is observed, which can be modeled as a game. To obtain spectrally efficient and fair spectrum allocation in this competitive environment with the nodes having no information on the other players, taxation of resources is applied to coerce desired behavior of the competitors. In the paper, we present mathematical formulation of the problem of finding the optimal taxation rate (common for all nodes) and propose a reduced-complexity algorithm for this optimization. Simulation results for these derived optimal values in various scenarios are also provided.
\end{abstract}

\section{Introduction}

Opportunistic spectrum access and flexible and efficient spectrum allocation procedures as well are considered as measures to increase the utilization of the scarce radio resources in future wireless communication networks. Apart from the spectral efficiency, the Quality of Experience (QoE), and the associated fairness in resources distribution are in the focus of research towards the cognitive, opportunistic, and dynamic spectrum access. The spectrum allocation procedures are usually centralized, require the Channel State Information (CSI) of all links in the network, and involve the overhead traffic, which in turn occupies the scarce radio resources. For the future communication concepts, such as cognitive or opportunistic radio, the nodes are expected to take intelligent decisions on the amount of resources to be utilized in a distributed way, thus minimizing or eliminating the overhead traffic.

In this paper, we consider opportunistic acquisition of orthogonal frequency channels by the network nodes. An example of the multiple-access technique using such orthogonal channels is the well-known Orthogonal Frequency Division Multiple Access (OFDMA). In the opportunistic
OFDMA, the network nodes are able to adopt a subset of accessible subcarriers (SCs) individually, as well as the transmission rate and power allocated to these SCs [1]. Below, we consider a more general scenario of the opportunistic access to frequency channels of any bandwidth, limited centralized management, and very limited control traffic, that is, there is no central frequency-channel scheduler, and no CSI exchange between the network nodes. Our approach to opportunistic spectrum allocation is related to noncooperative game theory and to the concept of pricing.

The game-theoretic scheduling for OFDMA has been considered in the literature as centralized and distributed SCs allocation. The centralized schemes allow for more efficient and fair spectrum utilization; however they require centralized management and a considerable amount of control traffic related to the CSI of all possible links in all considered frequency channels and to the information on the allocated channels. This information has to be exchanged or to be available at a central unit (e.g., at a base station of a cellular network) every time the channels qualities change for the nodes in the network area. Newest results for such centralized solutions based on cooperative complete information game models have been presented in [2-4]. Distributed decision 
making, on the contrary, deploys noncooperative games and seeks for Nash Equilibrium (NE) as a game solution. However, for the spectrum allocation, only the completeinformation games have been considered in the literature so far. We believe that such models cannot be considered for practical applications in dynamically changing wireless networks, since the complete knowledge of the CSI related to all links to be available at every other node would require a lot of control traffic between the nodes in dedicated control channels. This information would have to be sent every time the channels change, so in mobile environment, the control traffic would be comparable to the information-data traffic. Thus, noncooperative complete-information game models are only suitable for multicell environment, where the players are the base stations, which have the CSI of all links in their cell areas $[5,6]$, or in static wireless scenarios.

The concept of resource pricing (or coercive taxation) has been considered in the literature extensively for power allocation, for example, for OFDM and OFDMA in [79]. There, the resource that is taxed is the power used by the network nodes, and the goal is to maximize the sum throughput given the total allowable transmission power in the network. In these papers however, it has been assumed that the SCs distribution among the users has already been done somehow centrally.

Pricing has been also applied for distributed power and interference management in the network, for example, in [10] for the code-division multiple access. For this purpose the complete-information noncooperative game models have been formulated. Such game-theoretic problem formulation has practical application for interference management, due to the fact that the complete information on the interference level can be available for each player, since the nodes can measure it locally. However, for the spectrum management the problem is different.

Some papers, for example, [11-13] consider distributed allocation of resources based on pricing in a multicell scenario, where the base stations act as players. The pricing concepts developed for the multicell scenario, cannot be considered for the distributed resource allocation in decentralized networks, because contrary to a base station that may have the CSI of all links, a network node may only have the CSI of its own link. Another approach to price based spectrum management is based on iterative water filling, which allows all players to use the same frequency channels and adjust their power levels in these channels based on pricing function [14]. Definition of the pricing function for each player requires the CSI knowledge and exchange between the neighboring players, as well as the number of iterations. Similarly, in [15] the information exchange between the secondary and primary users is assumed for the spectrum leasing. In [16] the spectrum sharing is modeled as the oligopoly competition between the primary users and the Bertrand game model, which again requires the knowledge of the secondary users' CSI by the players (primary users). Although the above-mentioned works have contributed to significant advance in the game-theoretic price-based models for spectrum sharing, they all make an assumption on the complete information available for all players that relate to their links CSI or they narrow to the power and interference management.

In our earlier work [17], we have presented distributed SCs allocation interference-free method in a network of the OFDMA-based opportunistic radios. It aimed at rational and efficient spectrum utilization in both the uplink or downlink transmission. Rationality in our case means that apart from maximizing the spectral efficiency, the network and each individual node aim at lowering the cost of this efficiency (resulting from taxation of resources) and at increasing the QoE (resulting from the number of served nodes). These rationality measures have been reflected in the definition of the noncooperative game model with complete information, and in the utility function defined for each player (the network node). The definition of this game involved aggregation of the players, in such a way that each player (the network node) can view all other players as one, named the network-nodes community (NNC). The complete information required in this game does not include the individual CSI of the other network nodes, but only the local (single-link) CSI and the taxation rate. This way noncooperative game with full information is reasonable and practically applicable in the dynamically changing network scenarios.

Here, in this paper, we present a generalized framework for the taxation-based allocation of orthogonal frequency channels in the opportunistic radio. First, we show inappropriateness of the complete-information game models in the considered framework. Then, we consider selfish and social behavior of the players by appropriate definitions of the utility functions reflecting such behaviors. These utility functions include the linear-taxation summand dependent on the amount of the acquired spectrum resources. We aim at finding the optimal taxation rate to come up with a high overall network efficiency defined in two ways. We provide the mathematical description of the problem of finding the optimal tax rate, show that the problem complexity is NP hard, and present and examine the reduced-complexity algorithm for solving it.

In Section 2, we present the main idea of the proposed game-theoretic approach to distributed spectrum allocation and provide formal definitions of the considered games. In Section 3, we mathematically derive the amount of bandwidth each player is inclined to acquire. In Section 4, we present the reduced-complexity algorithm to obtain the optimal taxation, where the optimality is defined in a number of ways. The simulation results are presented in Section 5, and the work is concluded in Section 6.

\section{Taxation-Based Models of Distributed Spectrum Allocation}

We consider the scenery of multiple cognitive-radio nodes (or users) appearing in the opportunistic network area, that make use of the orthogonal frequency channels, for example, OFDMA subcarriers. It implies that the nodes do not have to apply any guard frequency bands to limit the out-of-band interference. The frequency correction at the 
receiver is also assumed to be perfect. This scenery of the opportunistic and cognitive radio network is presented in Figure 1. The nodes are able to sense the radio environment, detect the parameter called tax rate available in a given area, detect available spectrum resources, and acquire a subset of these resources usable for their intended transmission, for example, peer-to-peer communication, an access to a cellular network or to any wireless network in general. The goal of each node is to make the best use of these resources, that is, obtain high data rate at the lowest cost. As a proof of our concept, we consider the freedom in the spectrum allocation, that is, theoretically even the smallest part of the spectrum can be used by a player. This theoretical assumption can be refined for practical applications, if we assume that the nodes demand the spectrum only if they can make use of it, that is, if there is a minimal contiguous part of the spectrum available for their intended communication (such as one OFDM subcarrier band) that may include the protection band to mitigate the interference to other transmissions.

Let us consider the resource acquisition procedure as a game, which each network node plays against the other nodes (the players). Let us assume that there are $K$ players, and the available bandwidth is $B$. (For the simplicity of our considerations, in the remainder of this paper, we assume that $B$ and $K$ are fixed, although in dynamically changing network environment, the number of players, their demands, and the available bandwidth vary.) A single player decides what portion of the available bandwidth she is going to use. (Note that such a personification and female pronouns are established in the game-theoretic convention.) Selfish player aiming at her throughput maximization would occupy the whole available spectrum; however, such a behavior decreases the spectral efficiency and the capacity of the network, as well as the QoE of other players who cannot access the network. The problem is known as the Tragedy of Commons described in [18].

As a countermeasure for the problem of commons sharing and utilization, taxation of the resources is introduced. In our network scenery presented in Figure 1, the taxation rate is the same for all network nodes or users (the players). This tax rate is known in the considered area. It can be stored in an area database (among other parameters, required for the efficient operation of the cognitive or opportunistic users, e.g., the spectrum masks for available bands in a given location and time, primary-users detection thresholds, etc.) or transmitted by the elected master node in case, when the considered network operates independently from the area database in an ad hoc manner. It is being updated periodically and broadcasted in this area as one parameter among many other ones in a typical Broadcast Control CHannel (BCCH), or specifically defined Cognitive Pilot Channel (CPC) [19]. Let us stress that this broadcast transmission of a single parameter occupies really minor resources, contrary to the situation of transmitting full CSI of all links in the considered frequency band using dedicated channels.
2.1. Inappropriateness of the All Links Complete-Information Game Model. Let us first show that the completeinformation game model which makes use of the CSI of all involved links is not suitable for our scenario. The utility function for player $k$ in such a game, in which the concept of resource taxation is applied, reflects her throughput (revenue) and its related cost, and is defined as (Note that from this point, the mathematical analysis in this paper is performed in a continuous space for the sake of generality, however it can be easily translated into a discrete orthogonal channel scenario.)

$$
\varsigma\left(w_{k}(f)\right)=\int_{B_{1}}^{B_{2}} \log _{2}\left[1+\delta_{k} P_{k}(f) \gamma_{k}(f) w_{k}(f)\right] d f-r_{0} b_{k},
$$

where $w_{k}(f)$ is the function indicating the occupancy of the frequencies by player $k\left(w_{k}(f)=1\right.$ if frequency $f$ is assigned to user $k$ and $w_{k}(f)=0$ otherwise), $B_{1}$ and $B_{2}$ are the lower and the upper bounds of the available spectrum $\left(B_{2}-B_{1}=B\right), b_{k}$ is the amount of bandwidth the player acquires, $\gamma_{k}(f)=\left|H_{k}(f)\right|^{2} / \mathcal{N}_{0}$ is the Carrier-to-Noise Ratio (CNR) measured at the frequency $f, H_{k}(f)$ and $P_{k}(f)$ are the $k$ th user's channel characteristic and the power spectral density allocated to this frequency, respectively, and $\mathcal{N}_{0}$ is the noise power spectral density. Let us note that for the case of orthogonal channels, interference that is usually added to noise equals zero. Moreover, in (1), $\delta_{k}$ is the factor (often called the Signal-to-Noise Ratio (SNR)-gap) depending on the assumed player's Bit Error Probability (BEP) $P_{e_{k}}$. (In case of M-QAM, $\delta_{k}=-1,5 / \ln \left(0,5 P_{e_{k}}\right)$, while for $M \geq 4$ and the SNR in the range of $0-30 \mathrm{~dB}$ it can be set more precisely as $\delta_{k}=-1,5 / \ln \left(5 P_{e_{k}}\right)$ [20].) Finally, in the above equation, $r_{0}$ is a linear tax rate.

Since every channel can be used by a sole player:

$$
\begin{gathered}
\forall f \exists ! k: w_{k}(f)=1, \\
b_{k}=\int_{B_{1}}^{B_{2}} w_{k}(f) d f .
\end{gathered}
$$

To find the NE in such a game, we shall be solving this problem numerically. This numerical representation of finding the NE is the binary linear programming problem, that is, for a given set of $\gamma_{k}(f)$ values for all $K$ nodes and for a considered number of available channels we shall find binary values $w_{k}(f)$ for a given $r_{0}$. Then, looking for optimum $r_{0}$ to maximize some goal function, for example, the network sum throughput, would be even more complex. Note, that solution of such a defined problem would require the knowledge of all $\gamma_{k}(f)$ by every node, and thus, as mentioned before such a model for resource allocation is not practical.

2.2. Game Models Using Only the Local CSI. To narrow the space of this analysis and to eliminate the necessity for complete information concerning other players' strategies and payoffs, we propose to treat the rest of the players as a whole (the NNC). Note that NNC is not formally organized 


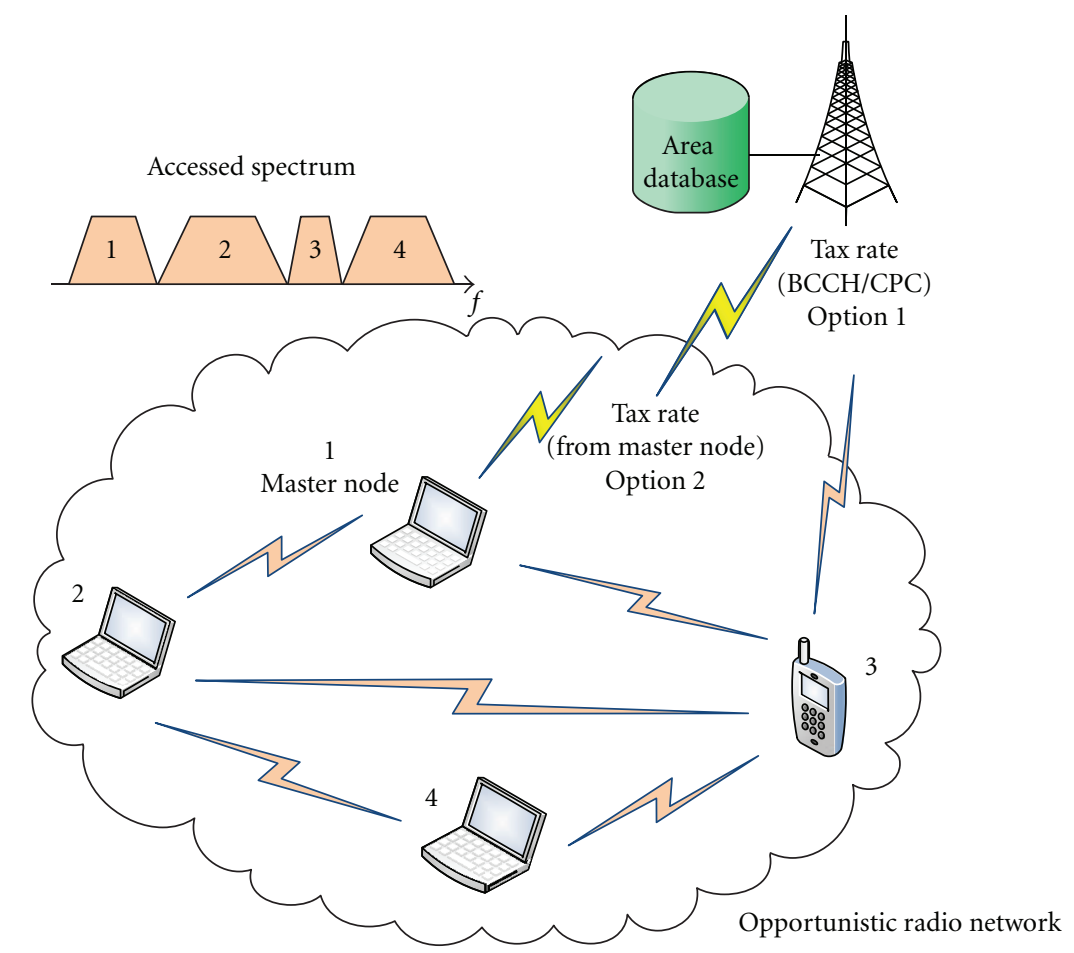

FIGURE 1: The scenery of the cognitive radio network with the taxation-based opportunistic spectrum access.

in any way. It is only viewed as such by a single player. Moreover, we let the players take decisions independently and subsequently one after another, as they appear in the network. It is a usual case in all wireless networks that some collision avoidance mechanism is implemented, or an access to decision-making entity (e.g., base-station) makes use of the random access channel to avoid taking decisions at the same time. We also limit the players in the maximum amount of bandwidth they can take at a time as a countermeasure for their greedy behavior, and let this maximum allowed bandwidth be $B_{I}$. The rationale behind such a limitation is proved in [21].

Let us first look at the utility defined for the $k$ th player so, as to reflect the player's throughput:

$$
\zeta_{k}\left(b_{k}\right)=\int_{f \in S_{k}} \log _{2}\left[1+\delta_{k} P_{k}(f) \gamma_{k}(f)\right] d f-r_{0} b_{k},
$$

where $\boldsymbol{S}_{k}$ is the (noncountable) set of frequencies player $k$ occupies. Let us note that before a decision is taken by player $k$, she senses the available spectrum resources and knows which frequency bands are already occupied (the amount of available bandwidth for the $k$ th player is $B_{k}$, and there is no possibility that different players acquire the same frequencies: $\left.\forall k \neq \mathrm{j}: S_{k} \cap S_{j}=\varnothing\right)$. Thus, the game model is dynamic. Moreover, the game for each player is two-dimensional only, what noticeably simplifies the problem.

The model defined above is not really a commonly understood game, since the decision of the NNC is not represented in the utility function (unless we call it a game against the radio environment, or a game against the nature as in [22]). This model reflects selfish behavior of the players, who care only for their own throughput. In the reminder of the paper we will call this model Selfish-Behavior Model.

In [17] we have proposed a noncooperative completeinformation two-dimensional game, where the payoffs of both players (player $k$ and the NCC at the $k$ th game stage) reflect their decisions. In such a case, being the alternative to the framework discussed above, the strategy space of the considered $k$ th player is the same (consists of possible number of the orthogonal channels the player acquires). The strategies of the NNC are the numbers of channels this community may occupy all together apart from the considered player. The utility function of the considered $k$ th player is defined as follows:

$$
\begin{aligned}
& \xi_{k}\left(b_{k}, c_{k}\right)=\left\{\frac{1}{B} \int_{f \in \mathrm{S}_{k}} \log _{2}\left[1+\delta_{k} P_{k}(f) \gamma_{k}(f)\right] d f\right\} \\
& \cdot\left\{B_{k}-b_{k}-c_{k}\right\}-r_{0} b_{k} \text {, }
\end{aligned}
$$

where $c_{k}$ is the amount of bandwidth that will be occupied by NNC, and $B_{k}$ is the amount of bandwidth available at the $k$ th game stage. One may interpret formula (4) as the total normalized throughput (throughput per frequency unit of the total available bandwidth $B$ ) which could be obtained by the new incoming players in case they occupied the remaining bandwidth and had the same average spectral efficiency as the considered player. This way, in the decision making on how much of the spectrum to occupy, the players factor the social aspect of the network (to serve multiple nodes) and not just their own benefit. In the reminder of the paper the game model with the $k$ th player's utility function defined by (4) will be called the Social-Behavior Model. 
Finally, the payoff for the NNC at the $k$ th game stage can be defined as $\varepsilon_{k}=c_{k}$, where $c_{k} \in\left[0, \max \left(0, B_{k}-B_{I}\right)\right]$, that is, the number of resources that can be potentially occupied by NNC.

\section{Optimal Choice of Spectrum Resources: The Players' Perspective}

Let us now consider how the players choose their strategic options, and how to coerce their desired behavior to obtain specifically defined network benefit.

3.1. Selfish-Behavior Model. As the practical approach to the Selfish-Behavior Model (SelBM) described by the utility function (3), we propose to eliminate dominated (disadvantageous) strategies of the players. Taking the $k$ th player's strongest frequencies (the ones that have the highest $\gamma_{k}(f)$ values) into account is in fact the elimination of dominated strategies of the considered player. Note, that from both the individual node and the whole network perspective, making use of the strongest channels results in higher spectral efficiency. Thus, the strategies of a single node are all possible channels of the strongest frequencies (from 0 to $B_{I}$ ). In order to find the optimum taxation, let us consider rewriting formula (3):

$$
\dot{\zeta}_{k}\left(b_{k}\right)=\int_{0}^{b_{k}} \log _{2}\left[1+\delta_{k} \dot{P}_{k}\left(f_{k}\right) \dot{\gamma}_{k}\left(f_{k}\right)\right] d f_{k}-r_{0} b_{k},
$$

where $\dot{\gamma}_{k}\left(f_{k}\right)$ is the function resulting from ordering (in the descending manner) of the continuous values of $\gamma_{k}(f)$ : (Ordering operation described by (6) is a hypothetical bijective mapping, which cannot be generically defined for any continuous space of values and depends on $\gamma_{k}(f)$. This operation involves mapping of both the domain arguments and the codomain images of $\gamma_{k}(f)$ to new arguments and images of $\dot{\gamma}_{k}\left(f_{k}\right)$ belonging to the same domain and codomain, respectively. For a discrete set of values ordering can be done by a standard sorting algorithm.)

$$
\dot{\gamma}_{k}\left(f_{k}\right) \longleftrightarrow \gamma_{k}(f): \forall n, m f_{k_{n}} \leq f_{k_{m}} \Longrightarrow \dot{\gamma}_{k}\left(f_{k_{n}}\right) \geq \dot{\gamma}_{k}\left(f_{k_{m}}\right) \text {, }
$$

and $\dot{P}_{k}\left(f_{k}\right)$ is the power spectral density resulting from the optimal power allocation for a total-power constraint, that is, from the water filling. Note that this ordering takes place at every stage, so in (5), the lower integration endpoint is always equal to zero. Moreover, as mentioned before

$$
b_{k} \leq \min \left\{B_{I}, B_{k}\right\},
$$

where $B_{\mathrm{I}}$ is the maximum allowable bandwidth one player can take at a time, and $B_{k}$ is the bandwidth available at the $k$ th game stage (for the $k$ th player). We also limit our choices of $b_{k}$ to only useful frequencies, that is,

$$
b_{k} \leq B_{\mathrm{WF}_{k}},
$$

where $B_{\mathrm{WF}_{k}}$ is the useful bandwidth after the water-filling, that is, the bandwidth, in which: $\dot{P}_{k}\left(f_{k}\right)>0$ for all $f_{k} \in$ $\left[0, B_{\mathrm{WF}_{k}}\right]$. In such a case:

$$
\forall f_{k} \leq b_{k}: \dot{P}_{k}\left(f_{k}\right)=\frac{1}{\gamma_{0 k}\left(b_{k}\right)}-\frac{1}{\delta_{k} \dot{\gamma}_{k}\left(f_{k}\right)},
$$

where $1 / \gamma_{0 k}\left(b_{k}\right)$ is the water level obtained in the water-filling algorithm over the acquired bandwidth $b_{k}$ of player $k$. The utility function $\dot{\zeta}_{k}\left(b_{k}\right)$ can be thus expressed as:

$$
\dot{\zeta}_{k}\left(b_{k}\right)=\int_{0}^{b_{k}} \log _{2}\left[\delta_{k} \dot{\gamma}_{k}\left(f_{k}\right) / \gamma_{0 k}\left(b_{k}\right)\right] d f_{k}-r_{0} b_{k} .
$$

It can be easily shown that the above-defined function is concave, (For all $b_{k}$ defined by (7) and (8), the first summand is concave and monotonically increasing because $\delta_{k} \dot{\gamma}_{k}\left(f_{k}\right) / \gamma_{0 k}\left(b_{k}\right)>1$ for all $f_{k}$, and the second summand is linearly decreasing with $b_{k}$ ) so we may find its maximum (as each rational player would do) by solving the following equation:

$$
\frac{\partial}{\partial b_{k}} \dot{\zeta}_{k}\left(b_{k}\right)=0
$$

As derived in Appendix A (formula (A.10)):

$$
\frac{\partial}{\partial b_{k}} \dot{\zeta}_{k}\left(b_{k}\right)=\mathscr{F}_{k}\left(b_{k}\right)-r_{0}
$$

where $\mathcal{F}_{k}\left(b_{k}\right)$ can be defined in a number of ways depending on the player's CNR characteristic $\gamma_{k}(f)$, and its resulting sorted values at the $k$ th game stage $\dot{\gamma}_{k}\left(f_{k}\right)$. As derived in Appendix A in (A.11) for the two-path propagation model it can be approximated as

$$
\begin{aligned}
\mathcal{F}\left(b_{k}\right) \simeq & \log _{2}\left\{A_{k} \delta_{k}\left[1+\cos \left(2 \pi b_{k} \tau_{k}-\varphi_{k}\right)\right]\right\} \\
& +\log _{2}\left\{b _ { k } ^ { - 1 } \left[\Gamma_{k}+\frac{1}{2 \pi \tau_{k} A_{k} \delta_{k}}\left[\operatorname{tg}\left(\pi b_{k} \tau_{k}-\frac{\varphi_{k}}{2}\right)\right.\right.\right. \\
& \left.\left.\left.-\operatorname{tg}\left(\frac{\varphi_{k}}{2}\right)\right]\right]\right\}+\frac{b_{k}}{\ln 2} \\
& \times\left\{\Gamma_{k}+\frac{1}{2 \pi \tau_{k} A_{k} \delta_{k}}\left[\operatorname{tg}\left(\pi b_{k} \tau_{k}-\frac{\varphi_{k}}{2}\right)-\operatorname{tg}\left(\frac{\varphi_{k}}{2}\right)\right]\right\}^{-1} \\
& \cdot\left\{2 A_{k} \delta_{k} \cos ^{2}\left(\pi b_{k} \tau_{k}-\frac{\varphi_{k}}{2}\right)\right\}^{-1},
\end{aligned}
$$

where the $A_{k}, \varphi_{k}$, and $\tau_{k}$ are the parameters of the considered multipath propagation model depending on the signal attenuation, average phase difference between the arriving multipath signal components, and the multipath delay spread (see Appendix A). Thus, by solving (11) we obtain the amount of bandwidth $b_{k}^{*}$ the $k$ th player is inclined to acquire

$$
b_{k}=b_{k}^{*}: \mathcal{F}\left(b_{k}^{*}\right)=r_{0} .
$$

Note, that for a given $r_{0}$, user $k$ can find $b_{k}^{*}$, not knowing other players CSI, and this finding is independent from other players' choices. 
3.2. Social-Behavior Model. Let us now consider formula (4) reflecting the Social Behavior Model (SocBM) in the form with ordered values of $\gamma_{k}(f)$ (similarly as in the previous section):

$$
\begin{aligned}
\dot{\xi}_{k}\left(b_{k}, c_{k}\right)=\frac{1}{B} & \left\{\int_{0}^{b_{k}} \log _{2}\left[1+\delta_{k} \dot{P}_{k}\left(f_{k}\right) \dot{\gamma}_{k}\left(f_{k}\right)\right] d f_{k}\right\} \\
\cdot & \left\{B_{k}-b_{k}-c_{k}\right\}-r_{0} b_{k},
\end{aligned}
$$

Based on (6)-(9), the above formula can be easily (again similarly as in the previous section) converted to

$$
\begin{aligned}
\dot{\xi}_{k}\left(b_{k}, c_{k}\right)= & \frac{1}{B}\left\{\int_{0}^{b_{k}} \log _{2}\left[\delta_{k} \dot{\gamma}_{k}\left(f_{k}\right) / \gamma_{0 k}\left(b_{k}\right)\right] d f_{k}\right\} \\
& \cdot\left\{B_{k}-b_{k}-c_{k}\right\}-r_{0} b_{k} .
\end{aligned}
$$

It can be shown that function (16) is concave, (For all $b_{k}$ defined by (7) and (8), the first factor in the first summand is concave and monotonically increasing, the second factor in the first summand is linearly decreasing with $b_{k}$, and so is the second summand.) so we may find its maximum (as each rational player would do) by solving the following equation:

$$
\frac{\partial}{\partial b_{k}} \dot{\xi}_{k}\left(b_{k}, c_{k}\right)=0
$$

The derivative $\left(\partial / \partial b_{k}\right) \dot{\xi}_{k}\left(b_{k}, c_{k}\right)$ is defined by formula (B.1) obtained in Appendix B, whose simplified form is the following:

$$
\frac{\partial}{\partial b_{k}} \dot{\xi}_{k}\left(b_{k}, c_{k}\right)=g_{k}\left(b_{k}, c_{k}\right)-r_{0}
$$

where $g_{k}\left(b_{k}, c_{k}\right)$ can be defined as in (B.3) for rural areas. (We do not repeat its long formula here. See Appendix B for its definition.) Thus, by solving (16) we obtain the amount of bandwidth $b_{k}^{*}$ which the $k$ th player is inclined to acquire taking into account the considered amount of bandwidth to be occupied by the NNC at the $k$ th game stage:

$$
b_{k}\left(c_{k}\right)=b_{k}^{*}\left(c_{k}\right): g\left(b_{k}^{*}\right)=r_{0} .
$$

In other words, $b_{k}^{*}\left(c_{k}\right)$ is the best-response function in the considered two-dimensional game.

Let us recall that the payoff of the NNC is defined as $\varepsilon_{k}=c_{k}$, where $c_{k} \in\left[0, \max \left(0, B_{k}-\mathrm{B}_{I}\right)\right]$ and is not dependent on the strategy of the $k$ th player $b_{k}$. Thus, the NNC would always choose the strategy $c_{k}^{*}=\max \left(0, B_{k}-\right.$ $\left.B_{I}\right)$ resulting in its highest possible payoff. For this NNC strategy $\left(c_{k}^{*}\right)$ and for $b_{k}^{*}\left(c_{k}^{*}\right)$ we obtain the NE. Thus, for the calculated equilibrium strategies the players acquire a portion of bandwidth for their transmission.

\section{Optimal Tax Rates}

To obtain the desired behavior of the players and high overall network efficiency, the tax rate for the considered games (presenting either the SelBM or SocMB) should be properly chosen to obtain the maximum benefit for the whole network in the considered framework, for example, the maximum sum throughput reflecting the efficiency of the spectrum distribution. We can define our objective function as

$$
\eta_{\mathrm{ST}}=\frac{1}{B} \sum_{k=0}^{K-1} \int_{0}^{b_{k}^{*}} \log _{2}\left[\delta_{k} \dot{\gamma}_{k}\left(f_{k}\right) / \gamma_{0 k}\left(b_{k}^{*}\right)\right] d f_{k},
$$

which is the sum throughput (ST) of all players averaged over the total available bandwidth $B$. Alternatively, we may look at maximizing the actual spectral efficiency (SE) of the transmission in the network (the sum throughput averaged over the actually used frequency bandwidth):

$$
\eta_{\mathrm{SE}}=\frac{1}{\sum_{k=0}^{K-1} b_{k}^{*}} \sum_{k=0}^{K-1} \int_{0}^{b_{k}^{*}} \log _{2}\left[\delta_{k} \dot{\gamma}_{k}\left(f_{k}\right) / \gamma_{0 k}\left(b_{k}^{*}\right)\right] d f_{k}
$$

The next step would be to find the optimum value of $r_{0}$ to maximize either function (20) or (21). Note that many other definitions of the objective function are possible, that could reflect the fairness or proportional fairness in the distribution of resources, as well as other factors, for example, the percentage of used bandwidth or the percentage of served players. Below, we will examine the two objective functions defined above by (20) and (21) and show that some fairness in resource distribution is also achieved with a taxrate optimal for (20).

The values of $\eta_{\mathrm{ST}}$ or $\eta_{\mathrm{SE}}$ depend on $r_{0}$, and on the values of $\dot{\gamma}_{k}\left(f_{k}\right)$. (This is because $r_{0}$ and $\dot{\gamma}_{k}\left(f_{k}\right)$ have the implication on $b_{k}^{*}$ and on the throughput obtained by player $k$.) Moreover, the order of appearance of the players in the game matters, since $\dot{\gamma}_{k}\left(f_{k}\right)$ depends on $\dot{\gamma}_{j}\left(f_{j}\right)$ for all $j<$ $k$ (frequencies allocated to players taking decisions before player $k$ must be excluded from this player considerations). Unfortunately, both $\eta_{\mathrm{ST}}$ and $\eta_{\mathrm{SE}}$ are neither strictly concave or convex functions of $r_{0}$. In general, for low $r_{0}$ it pays off for all players to acquire the highest possible amount of bandwidth, irrespective to their channel qualities. As $r_{0}$ increases, it becomes affordable to acquire some bandwidth only for the players with good channel conditions (high $\gamma_{k}(f)$ and $\left.\dot{\gamma}_{k}\left(f_{k}\right)\right)$. Thus, in such a case, the spectral efficiency $\eta_{\mathrm{SE}}$ is increased, and the average sum-throughput $\eta_{\mathrm{ST}}$ may be decreased. However, when $r_{0}$ is too high, that is, close to the barrage tax rate, only very few players can afford some small portion of the frequency band, so a lot of available bandwidth is not used, and thus both the average sum-throughput $\eta_{\mathrm{ST}}$ and the spectral efficiency of the network $\eta_{\mathrm{SE}}$ are low. Thus, there exist some optimum values for $r_{0}$ to maximize either $\eta_{\mathrm{ST}}$ or $\eta_{\mathrm{SE}}$ :

$$
\begin{aligned}
& r_{0}=r_{\mathrm{ST}_{0}}^{*}: \eta_{\mathrm{ST}}\left(r_{\mathrm{ST}_{0}}^{*}\right)=\max \eta_{\mathrm{ST}}\left(r_{0}\right), \\
& r_{0}=r_{\mathrm{SE}_{0}}^{*}: \eta_{\mathrm{SE}}\left(r_{\mathrm{SE}_{0}}^{*}\right)=\max \eta_{\mathrm{SE}}\left(r_{0}\right) .
\end{aligned}
$$

However, it is not straightforward to determine these optimum values. (As mentioned before, $\eta_{\mathrm{ST}}$ and $\eta_{\mathrm{ST}}$ depend on $\dot{\gamma}_{k}\left(f_{k}\right)$ functions, which have different arguments $f_{k}$ for different players, and on the order of players' appearance.) 
To find this optimum, even numerically, is a complex NPhard problem, and the optimization procedure has to be performed every time the users' channels change. Some simplifications can be obtained in finding this optimum in a proper time span (not necessarily shorter than the coherence time of the tracked processes: $\gamma_{k}(f)$ ), because the value of the optimum in the next time instant should be found close to the optimum value in the previous time instant. For this purpose we may apply a method that actually traces the variations of the objective function $\left(\eta_{\mathrm{ST}}\right.$ or $\left.\eta_{\mathrm{SE}}\right)$ rather than the variations of the players' channel conditions.

The considerations presented in this section for continuous orthogonal channels can be easily translated to discrete orthogonal channels, that is, to the case of having a set of $N$ available channels (e.g., OFDM subcarriers) to be acquired by the players. In such a case, the integrations in (5), (10), (15), (16), (20), and (21) should be replaced by summations, and the value of $b_{k}$ and $b_{k}^{*}$ should be approximated by the discrete number of channels $i_{k}$ of a particular bandwidth $\Delta_{f}$ (with a particular resolution). Moreover, as shown in [17], there exists the NE for the discrete orthogonal channels (like in OFDMA). Below, for such a case of the available bandwidth discretization, we present the optimal tax-ratesearching algorithm with reduced complexity tracing the instantaneous variations of the network-objective function $\eta_{\text {ST }}$ around its maximal value.

Step 1. Initialize algorithm:

Determine $N$ available channels of $\Delta_{f}$ bandwidth,

Determine the range of $r_{0} \in\left[r_{\min }, r_{\max }\right]$,

Determine the increment of $r_{0}: \Delta_{r_{0}}$,

Determine acceptable value of $\eta_{\mathrm{ST}}: \eta_{\mathrm{ST}}$ min

Determine $K, B_{I}$, measure the players' $\gamma_{k}(f)$,

Determine the order of players' appearance.

Step 2. Find the $b_{k}^{*}$ values for all considered tax-rates $r_{0}$.

Step 3. Calculate $\eta_{\mathrm{ST}}$ for all $\mathrm{r}_{0}$.

Step 4. Find optimal tax-rate $r_{\mathrm{ST}_{0}}^{*}$ that maximizes $\eta_{\mathrm{ST}}$.

Loop 1:

Step L1.1. Monitor the network-objective function $\eta_{\mathrm{ST}}$.

Step L1.2. If $\eta_{\mathrm{ST}}<\eta_{\mathrm{ST} \mathrm{T}_{\min }}$, go to Loop 2,

else go to Loop 1.

Step 5. Update (increase) $r_{\mathrm{ST}_{0}}^{*}: r_{\mathrm{ST}_{+}}^{*}:=r_{\mathrm{ST}_{0}}^{*}+\Delta_{r_{0}}$,

Step 6. Calculate the resulting $\eta_{\mathrm{ST}_{+}}=\eta_{\mathrm{ST}}\left(r_{\mathrm{ST}_{+}}^{*}\right)$,

Step 7. If $\eta_{\mathrm{ST}_{+}}>\eta_{\mathrm{ST}}$, go to Step 9 else go to Loop 3.

Loop 2: Repeat

Step L2.1. $r_{\mathrm{ST}_{0}}^{*}:=r_{\mathrm{ST}_{+}}^{*}$,

Step L2.2. $\eta_{\mathrm{ST}}:=\eta_{\mathrm{ST}_{+}}$,
Step L2.3. $r_{\mathrm{ST}_{+}}^{*}:=r_{\mathrm{ST}_{0}}^{*}+\Delta_{r_{0}}$,

Step L2.4. Calculate $\eta_{\mathrm{ST}_{+}}=\eta_{\mathrm{ST}}\left(r_{\mathrm{ST}_{+}}^{*}\right)$,

Until $\eta_{\mathrm{ST}_{+}}>\eta_{\mathrm{ST}}$.

Step 8. Go to Step 12.

Step 9. Update (decrease) $r_{\mathrm{ST}_{0}}^{*}: r_{\mathrm{ST}_{-}}^{*}:=r_{\mathrm{ST}_{0}}^{*}-\Delta_{r_{0}}$,

Step 10. Calculate the resulting $\eta_{\mathrm{ST}_{-}}=\eta_{\mathrm{ST}}\left(r_{\mathrm{ST}_{-}}^{*}\right)$,

Step 11. If $\eta_{\mathrm{ST}}>\eta_{\mathrm{ST}}$, go to Loop 3

else go to Step 12.

Loop 3: Repeat

Step L3.1. $r_{\mathrm{ST}_{0}}^{*}:=r_{\mathrm{ST}_{-}}^{*}$,

Step L3.2. $\eta_{\mathrm{ST}}:=\eta_{\mathrm{ST}_{-}}$,

Step L3.3. $r_{\mathrm{ST}_{-}}^{*}:=r_{\mathrm{ST}_{0}}^{*}-\Delta_{r_{0}}$,

Step L3.4. Calculate $\eta_{\mathrm{ST}_{-}}=\eta_{\mathrm{ST}}\left(r_{\mathrm{ST}_{-}}^{*}\right)$,

Until $\eta_{\mathrm{ST}}>\eta_{\mathrm{ST}}$.

Step 12. If $\eta_{\mathrm{ST}} \geq \eta_{\mathrm{ST}}$ min

communicate new tax-rate and go to Loop 1, else Warn: "No tax-rate meeting the objectives".

As it will be shown in the next section, $\eta_{\mathrm{ST}}$ is more appropriate as the network-objective function for the fairness of resource distribution among the players in the case of both SelBM and SocBM, and always has a maximum when $B_{\mathrm{I}}$ is properly chosen (not too small) for a given $K$ and $B$. Analogous algorithm to the one presented below can be performed for searching the maximum of $\eta_{\mathrm{SE}}$. The presented algorithm has reduced complexity due to the application of the following methods: optimum tax-rate searching around the previous optimum and optimization procedure running only when $\eta_{\mathrm{ST}}$ drops below the required value: $\eta_{\mathrm{ST}}$ min .

Alternatively, to reduce the rate of necessary calculations to solve the optimization problem, we may maximize the expected values of (20) or (21) over the set of random variables $\dot{\gamma}_{k}\left(f_{k}\right): \bar{\eta}_{\mathrm{ST}}=\mathbb{E}_{\dot{\gamma}_{k}}\left\{\eta_{\mathrm{ST}}\right\}, \bar{\eta}_{\mathrm{SE}}=\mathbb{E}_{\dot{\gamma}_{k}}\left\{\eta_{\mathrm{SE}}\right\}$. Such definitions of the objective functions could be useful if we were able to approximate the expectation values with the average values and use them in a static (or slowly changing) environment. The resulting tax rate would approximate the optimum one (either $r_{\mathrm{ST}_{0}}^{*}$ or $r_{\mathrm{SE}_{0}}^{*}$ ) with unknown accuracy, while the optimization procedure can be performed off-line. This option is to be investigated in the future.

\section{Numerical Results}

Our simulation setup is the following. We have considered an available bandwidth $B$ with the resolution $\Delta_{f}=B / 256$, where $\Delta_{f}$ can be considered as the smallest spectrum unit, that can be occupied by orthogonal signals, for example, OFDM subcarriers. In our considered scenario, the total transmission power has been fixed. The power constraint for each link results from the distance between the transmitter 


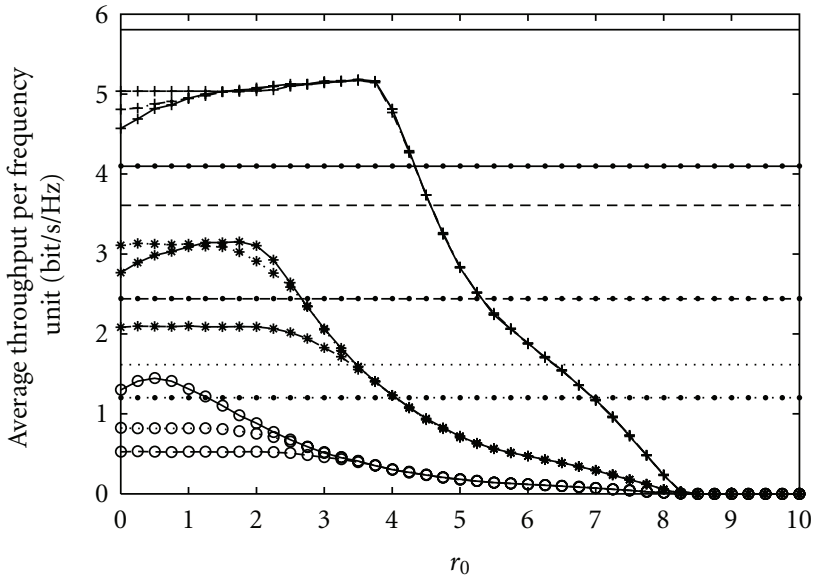

- Greedy alg., $K=64$

$\longrightarrow \mathrm{R}-\mathrm{R}, K=64$

- +- Tax., $K=64, B_{I}=B / 32$

… Tax., $K=64, B_{I}=B / 16$

, Tax., $K=64, B_{I}=B$

- - - Greedy alg., $K=16$

- R-R, $K=16$

-* - Tax., $K=16, B_{I}=B / 32$

*.. Tax., $K=16, B_{I}=B / 16$

$\rightarrow$ Tax., $K=16, B_{I}=B$

…. Greedy alg., $K=4$

…. R-R, $K=4$

- $\theta$ - Tax., $K=4, B_{I}=B / 32$

.... Tax., $K=4, B_{I}=B / 16$

$\rightarrow$ Tax., $K=4, B_{I}=B$

FIgURE 2: Average sum throughput $\eta_{\mathrm{ST}}$ versus the tax rate for SelBM; two-path channel model.

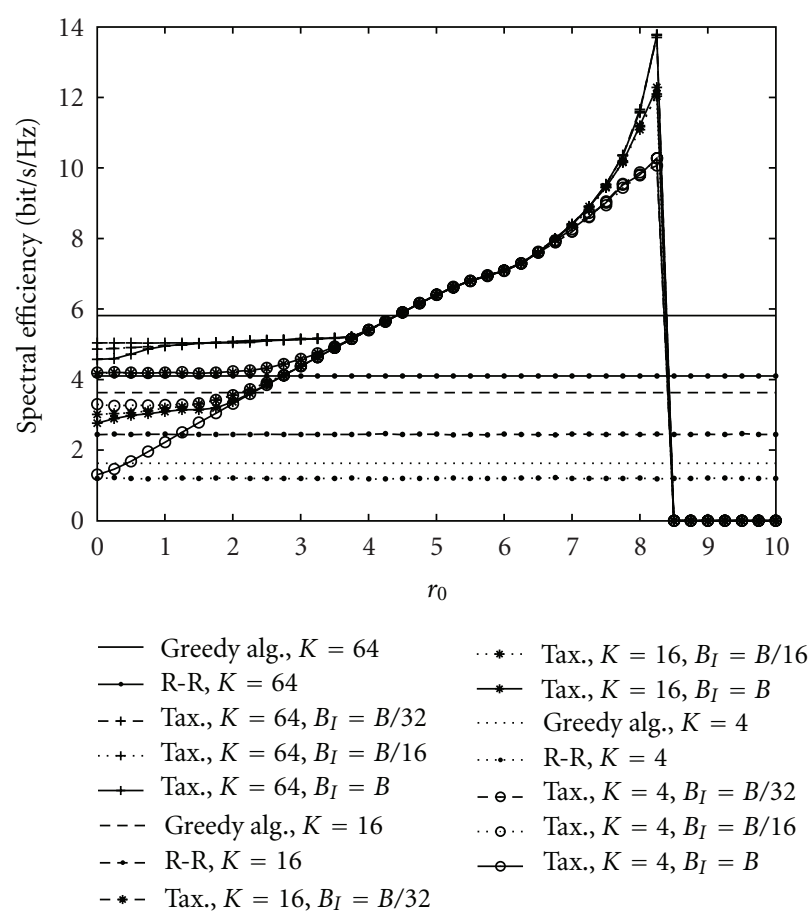

FIGURE 3: Spectral efficiency $\eta_{\mathrm{SE}}$ versus the tax rate for SelBM; twopath channel model.

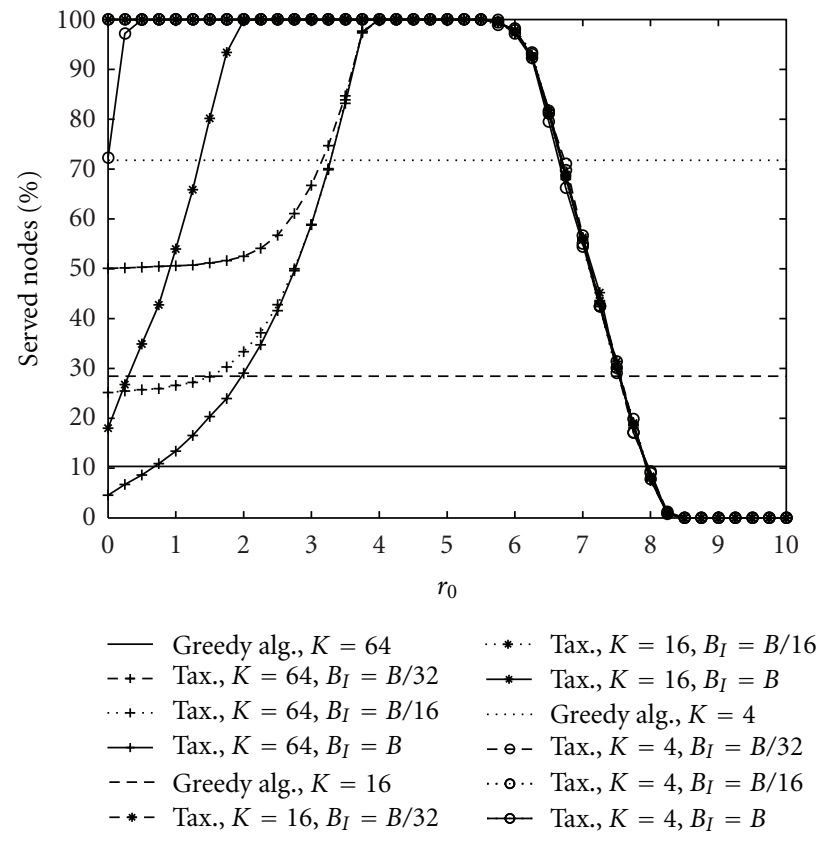

FIGURE 4: The percentage of served nodes versus the tax rate for SelBM; two-path channel model.

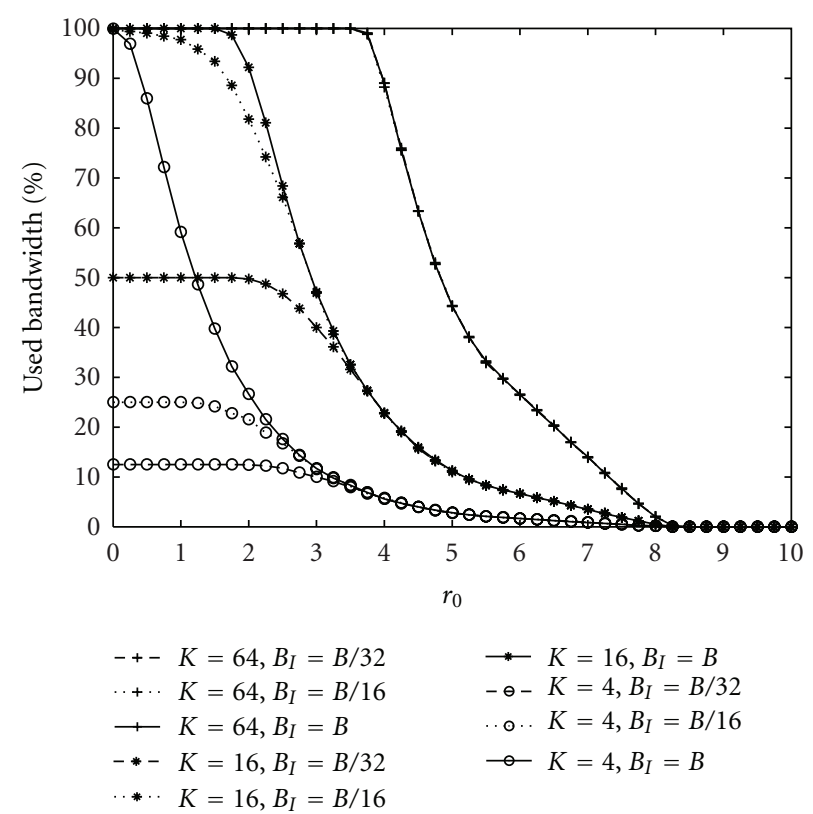

FIGURE 5: The percentage of used bandwidth versus the tax rate for SelBM; two-path channel model.

and the receiver and from the power-control mechanism. (Usually this mechanism is applied to combat the nearfar effect and the interference between the users; however, here, we assume orthogonal frequency channels, so this mechanism is only used to assure the appropriate quality of the link, i.e., the required average SNR, which in our case has been set to $30 \mathrm{~dB}$ ). For our simulation purposes, it has 


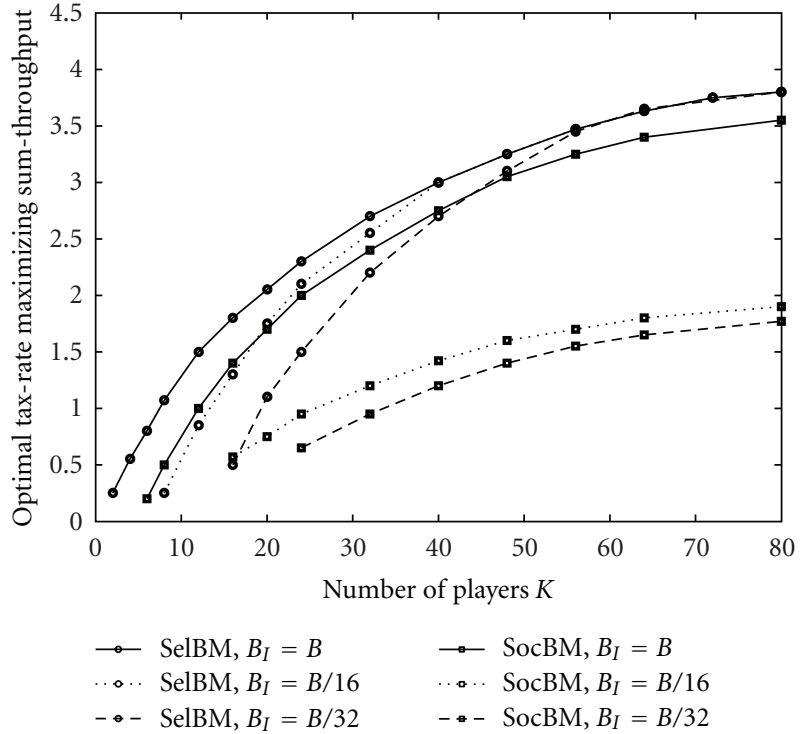

FIgURE 6: Optimal tax rate for sum throughput $r_{\mathrm{ST}_{0}}^{*}$ versus the number of competing players for the SelBM and the SocBM; twopath channel model.

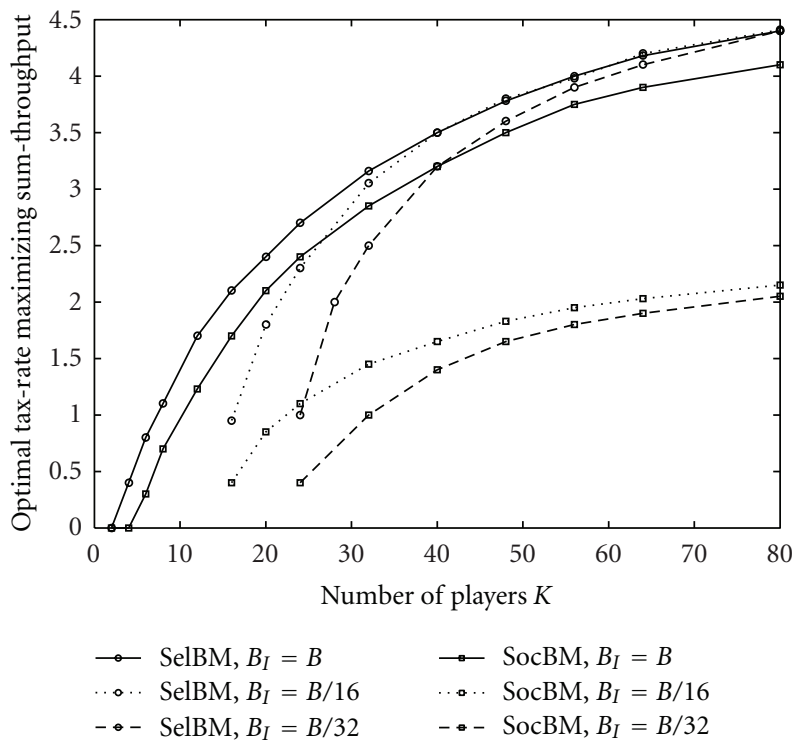

FIGURE 7: Optimal tax rate for sum throughput $r_{\mathrm{ST}_{0}}^{*}$ versus the number of competing players for the SelBM and the SocBM; sixpath channel model.

been assumed that the order of appearance of the players in a game is random. Furthermore, we assume that the power control mechanism has a tolerance of $3 \mathrm{~dB}$, so that random deviation from the average SNR is possible for any node (average SNR $=(30 \pm 3) \mathrm{dB})$. This average SNR deviation (which also reflects the accuracy of power-control in modern radio systems) has been chosen to differentiate possible link qualities. Moreover, two example channel models have

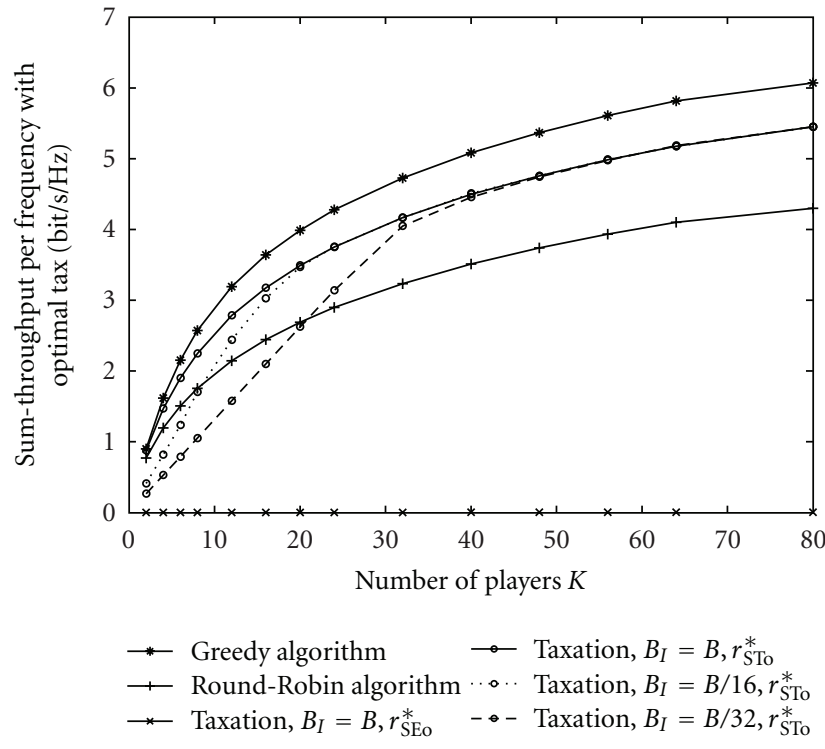

FIGURE 8: Average sum throughput for the optimal tax rate for both SelBM and SocBM; two-path channel model.

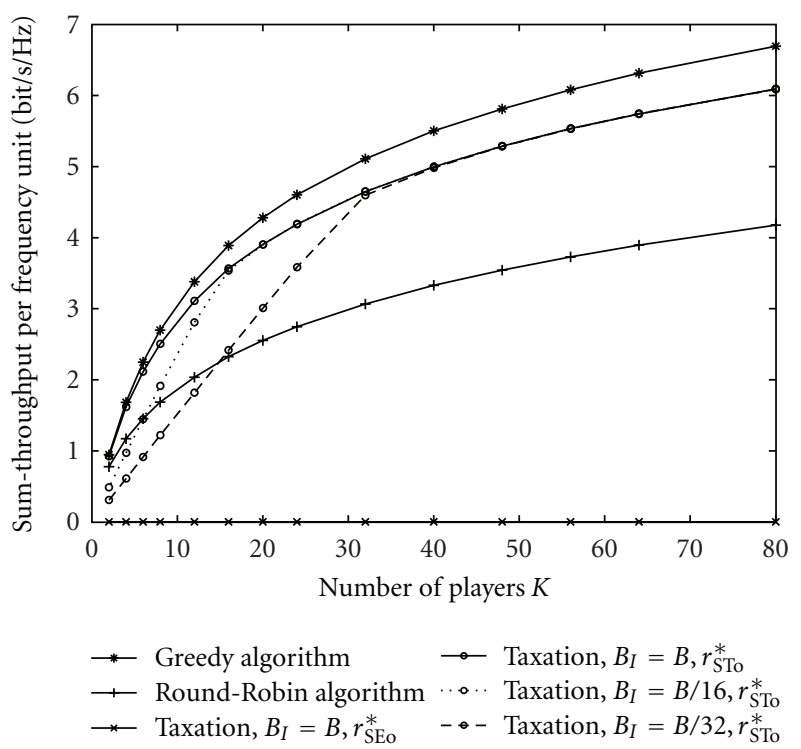

FIGURE 9: Average sum throughput for the optimal tax rate for both SelBM and SocBM; six-path channel model.

been compared. The first one is the two-path Rayleighfading channel with the delay spread ranging from 0 to $1 / 64$ of $1 / B$, and the average power of the second path being $-3 \mathrm{~dB}$ relative to the first path (such a model can be considered as suitable for rural environment). The second considered model is the six-path channel, with paths having the same power, and delays uniformly spread between 0 to $1 / B$. (This is a test-channel model often used for the test of equalizers that reflects particularly hostile environment with very small coherence bandwidth and very deep fading.) We 


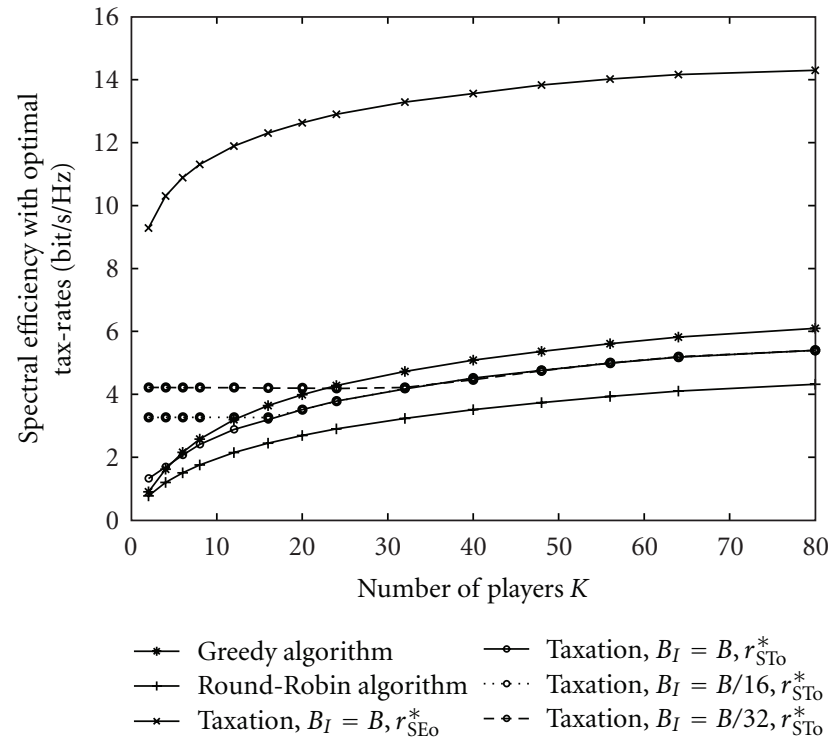

Figure 10: Spectral efficiency for the optimal tax rate for both SelBM and SocBM; two-path channel model.

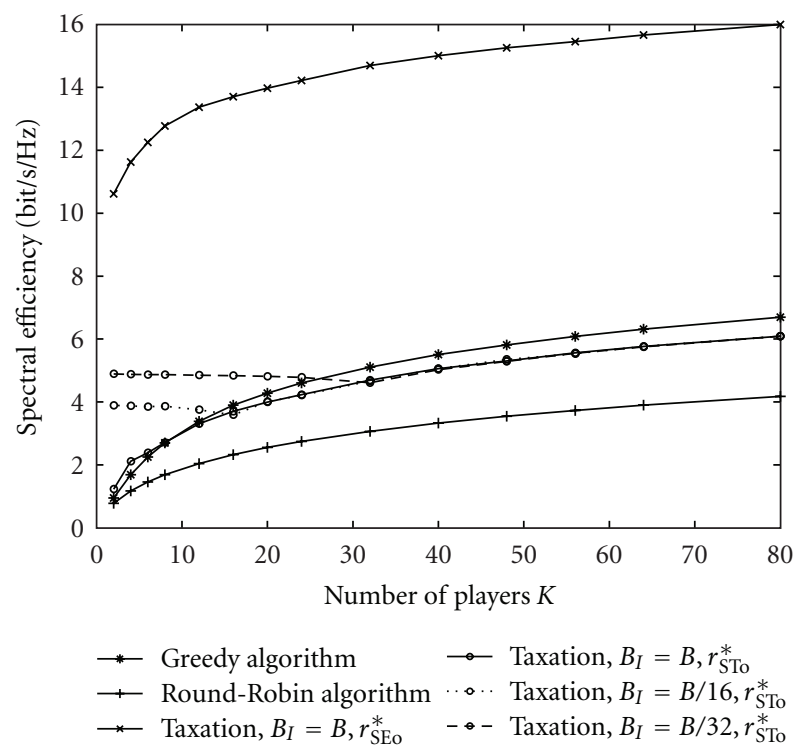

FIGURE 11: Spectral efficiency for the optimal tax rate for both SelBM and SocBM; six-path channel model.

have observed 1000 channel realizations and assumed the target BEP $P_{e_{k}}=10^{-3}$ for all links (for all $k$ ).

For the comparison purposes, we present results of our proposed framework and the reduced-complexity algorithm of finding the optimal tax rate together with the results of the greedy algorithm (that assigns the frequencies to the players with the highest $\mathrm{CNR}$ values at these frequencies) and Round-Robin algorithm of resource distribution. Although both of these algorithms can be only implemented in a centralized manner, they give the two opposite extremes: either maximum spectral efficiency or maximum fairness for the case of the whole used bandwidth.

Let us first analyze the network performance in the case of the users' SelBM and the influence of the tax rate $r_{0}$, the restricted amount of bandwidth $B_{\mathrm{I}}$, and the number of players $K$ on the network behavior. In Figures 2 and 3 we observe the averaged sum throughput $\eta_{S T}$ defined by (20) and the network spectral efficiency $\eta_{\mathrm{SE}}$ defined by (21), respectively, for the two-path channel model. As we can see, there is some optimum tax rate $r_{\mathrm{ST}_{0}}^{*}$ that maximizes $\eta_{\mathrm{ST}}$ when $B_{\mathrm{I}}$ is not too small for a given $K$. Otherwise, $\eta_{\mathrm{ST}}$ is constant for low tax rates, and then, for higher tax rates decreases to zero. The optimal tax rate $r_{\mathrm{SE}_{0}}^{*}$ that maximizes $\eta_{\mathrm{SE}}$ is close to the barrage tax.

To better understand the mechanism of increasing tax rates in the network, already discussed in the previous section, let us analyze Figures 4 and 5 . There, the percentage of served nodes and the percentage of used bandwidth are shown versus the tax rate $r_{0}$. (We assume that the node is served if it is able to acquire a portion of bandwidth satisfying her target BEP.) As we can see, low taxes allow to utilize most of the bandwidth and serve most of the users, again when $B_{\mathrm{I}}$ is properly chosen. In general, restricting the players in the amount of bandwidth they can take at their turn has negative influence on $\eta_{\mathrm{ST}}$ (the maximum is always achieved for $B_{I}=B$ ) and on the percentage of used bandwidth, and positive influence on $\eta_{\mathrm{SE}}$ and the percentage of served nodes, but only for relatively low tax rates. For higher tax rates, for which $\eta_{\mathrm{ST}}$ and the percentage of used bandwidth dramatically drop, both $\eta_{\mathrm{SE}}$ and the percentage of served nodes are not dependent on $B_{I}$. Thus, our first conclusion is that for the fairness of the resource distribution, it is better to apply $B_{I}=B$, and calibrate just the tax rate to optimize $\eta_{\mathrm{ST}}$ rather than $\eta_{\mathrm{SE}}$. Similar (analogous) conclusions can be derived for the SocBM and for the other channel model.

In Figures 6 and 7 we can observe the tax rates $r_{\mathrm{ST}_{0}}^{*}$ optimizing $\eta_{\mathrm{ST}}$ for both the SelBM and the SocBM and in the case of two-path and six-path channel models. These tax rates have been found using the algorithm defined in the previous section. As we can see, for the SelBM the optimum tax rates $r_{\mathrm{ST}_{0}}^{*}$ for different $B_{I}$, converge to the same value as $K$ increases. It is not the case for the SocBM. Moreover, for the two-path channel model, the values of the optimum tax rate $r_{S_{0}}^{*}$ are higher than for the six-path channel model.

The tax-rate that optimizes $\eta_{\mathrm{SE}}$ does not depend on $K$. For the SelBM, it also does not depend on $B_{I}$, but only varies for different channel models. For the SocBM, $r_{\mathrm{SE}_{0}}^{*}$ depends on both, $B_{I}$ and the channel model. This can be observed in Table 1.

In Figures 8 and 9 one can observe the average sum throughput $\eta_{\mathrm{ST}}$ resulting from the optimal taxation versus the number of competing players $K$, for both the SelBM and the SocBM and in the case of two-path (Figure 8) and six-path (Figure 9) channel models. In Figures 10 and 11 the network spectral efficiency $\eta_{\mathrm{SE}}$ is presented versus $K$ for the same cases. Note, that for a given channel model the achievable average sum throughput $\eta_{\mathrm{ST}}$ is exactly the same for both behavior models: either SelBM or SocBM (although the respective optimal tax rates are different). The same 
TABLE 1: Optimal tax rates $r_{\mathrm{SE}_{0}}^{*}$ maximizing the network spectral efficiency $\eta_{\mathrm{SE}}$ for all values of $K$.

\begin{tabular}{lcccccc}
\hline Behavior & \multicolumn{3}{c}{ Two-path } & \multicolumn{3}{c}{ Six-path } \\
Model & $B_{I}=B$ & $B / 16$ & $B / 32$ & $B_{I}=B$ & $B / 16$ & $B / 32$ \\
\hline SelBM & 8.3 & 8.3 & 8.3 & 9.65 & 9.65 & 9.65 \\
SocBM & 8.2 & 4.35 & 4.25 & 9.6 & 5.05 & 4.9 \\
\hline
\end{tabular}

holds for the achievable spectral efficiency $\eta_{\mathrm{SE}}$. The difference between the plots occurs for different channel models. The achievable $\eta_{\mathrm{ST}}$ as well as $\eta_{\mathrm{SE}}$ are higher for the six-path channel model than for the two-path channel model due to the fact that this six-path model presents higher diversity in the subbands qualities for each player, so the players can make better choices. Finally, we have observed that when the optimum tax rate $r_{\mathrm{ST}_{0}}^{*}$ is applied in either scenario, $99-100 \%$ of nodes are served.

Note, that our framework cannot result in the maximal achievable sum throughput, which can be only obtained when the problem described by (1) is solved, which assumes complete-information of all links CSI and $K$-dimensional game that can be solved in either cooperative or noncooperative manner.

\section{Conclusions}

Here above, we have presented a game-theory-related framework for distributed allocation of spectrum resources in the opportunistic radio access networks. Contrary to the methods presented in the literature so far, in our game models, we do not assume the complete knowledge of the players CSI. Each player has the information on her own CSI only. Additionally, the taxation-rate parameter available in a data base and mandatory in the considered area is made known to the players through the broadcast channel (BCCH or the CPC). This significantly reduces the amount of control traffic in the network when compared with the frequent exchange of the all links CSI in the dedicated channels. Above, we have proposed a reduced-complexity algorithm of finding and tracing the optimum tax-rate value maximizing the network objective function. Our presented framework and the algorithm of finding the optimal taxation-rate result in high network benefit reflected in the sum throughput, but also in fairness of resource distribution (understood as the number of served nodes). The simulation results show that it is more beneficial for the network and for the individual players to use taxation with the tax rate maximizing the network sum throughput rather than to additionally limit the users in the maximum bandwidth they can acquire at the time. It is also more beneficial than maximization of the network spectral efficiency due to better utilization of the spectrum resources and higher percentage of served nodes. Simulation results also show that in the considered scenarios, when the optimal tax rate is applied the achievable sum throughput per frequency unit is as high as $5.5-6[\mathrm{bits} / \mathrm{s} / \mathrm{Hz}]$ (depending on the considered propagation model) for sufficiently high number of players. Moreover, in such a case, $99-100 \%$ of nodes are served in the network, that is, are able to acquire some resources satisfying their target BEP.

\section{Appendices}

A.

Below, we calculate the derivative of $\dot{\zeta}_{k}\left(b_{k}\right)$ :

$$
\begin{aligned}
\frac{\partial}{\partial b_{k}} \dot{\zeta}_{k}\left(b_{k}\right)= & \frac{\partial}{\partial b_{k}}\left\{\int_{0}^{b_{k}} \log _{2}\left[\delta_{k} \dot{\gamma}_{k}\left(f_{k}\right) / \gamma_{0 k}\left(b_{k}\right)\right] d f_{k}\right\} \\
& -\frac{\partial}{\partial b_{k}}\left\{r_{0} b_{k}\right\} \\
= & \frac{\partial}{\partial b_{k}}\left\{\int_{0}^{b_{k}} \log _{2}\left[\delta_{k} \dot{\gamma}_{k}\left(f_{k}\right)\right] d f_{k}\right\} \\
& -\frac{\partial}{\partial b_{k}}\left\{\int_{0}^{b_{k}} \log _{2}\left[\gamma_{0 k}\left(b_{k}\right)\right] d f_{k}\right\}-r_{0} .
\end{aligned}
$$

Because the one integrand in the first summand does not depend on $b_{k}$, and the one integrand in the second summand does not depend on $f_{k}$, further derivation of the above formula is the following:

$$
\begin{aligned}
\frac{\partial}{\partial b_{k}} \dot{\zeta}_{k}\left(b_{k}\right)= & \log _{2}\left[\delta_{k} \dot{\gamma}_{k}\left(b_{k}\right)\right] \\
& -\frac{\partial}{\partial b_{k}}\left\{b_{k} \log _{2}\left[\gamma_{0 k}\left(b_{k}\right)\right]\right\}-r_{0} \\
= & \log _{2}\left[\delta_{k} \dot{\gamma}_{k}\left(b_{k}\right)\right]-b_{k} \frac{\partial}{\partial b_{k}}\left\{\log _{2}\left[\gamma_{0 k}\left(b_{k}\right)\right]\right\} \\
& -\log _{2}\left[\gamma_{0 k}\left(b_{k}\right)\right]-r_{0} \\
= & \log _{2}\left[\delta_{k} \dot{\gamma}_{k}\left(b_{k}\right)\right]-\frac{1}{\ln 2} \frac{b_{k}}{\gamma_{0 k}\left(b_{k}\right)} \frac{\partial}{\partial b_{k}}\left\{\gamma_{0 k}\left(b_{k}\right)\right\} \\
& -\log _{2}\left[\gamma_{0 k}\left(b_{k}\right)\right]-r_{0} .
\end{aligned}
$$

Now, this formula does not have a closed form, that is, $\left(\partial / \partial b_{k}\right) \dot{\zeta}_{k}\left(b_{k}\right)$ depends on the particular shapes of $\dot{\gamma}_{k}\left(b_{k}\right)$ and of $\gamma_{0 k}\left(b_{k}\right)$ (where the later function in turn depends on the shape of the former). As an example, let us consider the rural area with the two-path propagation. In such a case, the channel power characteristic can be described as

$$
\gamma_{k}(f)=\left|\alpha_{k}\right|^{2}\left|1-\beta_{k} \exp \left\{-j\left(2 \pi f \tau_{k}-\varphi_{k}\right)\right\}\right|^{2},
$$

where $\alpha_{k}$ is the complex amplitude attenuation of the first path, $\beta_{k}$ is the attenuation of the second path relative to the first path, $\tau_{k}$ is the multipath delay spread, and $\varphi_{k}$ is average phase difference between the arriving multipath signal components. Let us denote $\phi_{k}=2 \pi f \tau_{k}-\varphi_{k}$. For the sufficient distance between the transmitting and receiving antennas $\beta_{k} \approx 1$. Consequently,

$$
\begin{aligned}
\gamma_{k}(f) & \simeq \alpha_{k}^{2}\left(1-\cos \phi_{k}+j \sin \phi_{k}\right)\left(1-\cos \phi_{k}-j \sin \phi_{k}\right) \\
& =2 \alpha_{k}^{2}\left(1-\cos \phi_{k}\right)=2 \alpha_{k}^{2}\left[1-\cos \left(2 \pi f \tau_{k}-\varphi_{k}\right)\right] .
\end{aligned}
$$


The above function is periodic and monotonically increasing for $\phi \in[0, \pi]$. Therefore, its sorted (in a descending order) version $\dot{\gamma}_{k}\left(f_{k}\right)$ can be approximated as

$$
\dot{\gamma}_{k}\left(f_{k}\right) \simeq A_{k}\left[1+\cos \left(2 \pi f_{k} \tau_{k}-\varphi_{k}\right)\right] \text {, }
$$

where $A_{k}$ is the proportionality constant.

Now, for our propagation model, let us find the formula for the water-level $\gamma_{0 k}\left(b_{k}\right)$ dependent on the shape of function $\dot{\gamma}_{k}\left(f_{k}\right)$ and on the considered bandwidth $b_{k}$. To this end we will integrate both sides of (9) resulting in

$$
\frac{b_{k}}{\gamma_{0 k}\left(b_{k}\right)}=\Gamma_{k}+\int_{0}^{b_{k}} \frac{1}{\delta_{k} \dot{\gamma}_{k}\left(f_{k}\right)} d f_{k}
$$

where $\Gamma_{k}$ is the power limit for player $k$. Consequently

$$
\gamma_{0 k}\left(b_{k}\right) \simeq b_{k}\left\{\Gamma_{k}+\int_{0}^{b_{k}} \frac{1}{A_{k} \delta_{k}\left[1+\cos \left(2 \pi f_{k} \tau_{k}-\varphi_{k}\right)\right]} d f_{k}\right\}^{-1},
$$

and because $\int\left(1 / \cos \phi_{k}\right) d \phi_{k}=\operatorname{tg} \phi_{k} / 2$, we obtain

$$
\begin{aligned}
& \gamma_{0 k}\left(b_{k}\right) \simeq b_{k}\left\{\Gamma_{k}+\frac{1}{2 \pi \tau_{k} A_{k} \delta_{k}}\right. \\
& {\left.\left[\operatorname{tg}\left(\pi b_{k} \tau_{k}-\frac{\varphi_{k}}{2}\right)-\operatorname{tg}\left(\frac{\varphi_{k}}{2}\right)\right]\right\}^{-1} . }
\end{aligned}
$$

Using the above expression we will derive the second term in (A.2):

$$
\begin{aligned}
\frac{b_{k}}{\gamma_{0 k}\left(b_{k}\right)} \frac{\partial}{\partial b_{k}}\left\{\gamma_{0 k}\left(b_{k}\right)\right\} \\
\simeq\left\{\Gamma_{k}+\frac{1}{2 \pi \tau_{k} A_{k} \delta_{k}}\left[\operatorname{tg}\left(\pi b_{k} \tau_{k}-\frac{\varphi_{k}}{2}\right)-\operatorname{tg}\left(\frac{\varphi_{k}}{2}\right)\right]\right\} \\
\cdot\left(-b_{k}\right)\left\{\Gamma_{k}+\frac{1}{2 \pi \tau_{k} A_{k} \delta_{k}}\right. \\
\left.\times\left[\operatorname{tg}\left(\pi b_{k} \tau_{k}-\frac{\varphi_{k}}{2}\right)-\operatorname{tg}\left(\frac{\varphi_{k}}{2}\right)\right]\right\}^{-2} \\
=\left(-b_{k}\right) \quad \\
\times\left\{\Gamma_{k}+\frac{1}{2 \pi \tau_{k} A_{k} \delta_{k}}\left[\operatorname{tg}\left(\pi b_{k} \tau_{k}-\frac{\varphi_{k}}{2}\right)-\operatorname{tg}\left(\frac{\varphi_{k}}{2}\right)\right]\right\}^{-1} \\
\cdot\left\{2 A_{k} \delta_{k} \cos ^{2}\left(\pi b_{k} \tau_{k}-\frac{\varphi_{k}}{2}\right)\right\}^{-1}
\end{aligned}
$$

We can now substitute (A.5)-(A.9) to (A.2), which results in

$$
\frac{\partial}{\partial b_{k}} \dot{\zeta}_{k}\left(b_{k}\right)=\mathcal{F}_{k}\left(b_{k}\right)-r_{0}
$$

where the first term on the right-hand side of the above equation $\mathcal{F}_{k}\left(b_{k}\right)$ does not depend on $r_{0}$, and for the considered channel model can be approximated as

$$
\begin{aligned}
\mathcal{F}_{k}\left(b_{k}\right) \simeq & \log _{2}\left\{A_{k} \delta_{k}\left[1+\cos \left(2 \pi b_{k} \tau_{k}-\varphi_{k}\right)\right]\right\} \\
& +\log _{2}\left\{b _ { k } ^ { - 1 } \left[\Gamma_{k}+\frac{1}{2 \pi \tau_{k} A_{k} \delta_{k}}\left[\operatorname{tg}\left(\pi b_{k} \tau_{k}-\frac{\varphi_{k}}{2}\right)\right.\right.\right. \\
& \left.\left.\left.-\operatorname{tg}\left(\frac{\varphi_{k}}{2}\right)\right]\right]\right\} \\
& \frac{b_{k}}{\ln 2}\left\{\Gamma_{k}+\frac{1}{2 \pi \tau_{k} A_{k} \delta_{k}}\left[\operatorname{tg}\left(\pi b_{k} \tau_{k}-\frac{\varphi_{k}}{2}\right)\right.\right. \\
& \times\left\{2 A_{k} \delta_{k} \cos ^{2}\left(\pi b_{k} \tau_{k}-\frac{\varphi_{k}}{2}\right)\right\}^{-1} \cdot
\end{aligned}
$$

The above formula can be further simplified, when we assume that the phase difference between the arriving twopath waveform components is negligible due to similar distance that both waves travel, that is, when $\varphi_{k} \approx 0$. Note, that formula (A.10) is very general, and function $\mathcal{F}\left(b_{k}\right)$ can be defined in a number of ways depending on the assumed propagation environment models.

\section{B.}

Below, we calculate the derivative of $\dot{\xi}\left(b_{k}, c_{k}\right)$ :

$$
\begin{aligned}
\frac{\partial}{\partial b_{k}} \dot{\xi}_{k}\left(b_{k}, c_{k}\right)= & \frac{\partial}{\partial b_{k}}\left\{\left[\int_{0}^{b_{k}} \log _{2}\left(\delta_{k} \dot{\gamma}_{k}\left(f_{k}\right) / \gamma_{0 k}\left(b_{k}\right)\right) d f_{k}\right]\right. \\
& \left.\times\left(B_{k}-b_{k}-c_{k}\right)\right\}-\frac{\partial}{\partial b_{k}}\left\{r_{0} b_{k}\right\} \\
= & \frac{\partial}{\partial b_{k}}\left\{\int_{0}^{b_{k}} \log _{2}\left(\delta_{k} \dot{\gamma}_{k}\left(f_{k}\right) / \gamma_{0 k}\left(b_{k}\right)\right) d f_{k}\right\} \\
& \times\left(B_{k}-b_{k}-c_{k}\right) \\
& -\int_{0}^{b_{k}} \log _{2}\left(\delta_{k} \dot{\gamma}_{k}\left(f_{k}\right) / \gamma_{0 k}\left(b_{k}\right)\right) d f_{k}-r_{0} \\
= & \frac{\partial}{\partial b_{k}}\left\{\int_{0}^{b_{k}} \log _{2}\left[\delta_{k} \dot{\gamma}_{k}\left(f_{k}\right)\right] d f_{k}\right\}\left(B_{k}-b_{k}-c_{k}\right) \\
& -\frac{\partial}{\partial b_{k}}\left\{\int_{0}^{b_{k}} \log _{2}\left[\gamma_{0 k}\left(b_{k}\right)\right] d f_{k}\right\} \\
& \cdot\left(B_{k}-b_{k}-c_{k}\right) \\
& -\int_{0}^{b_{k}} \log _{2}\left(\delta_{k} \dot{\gamma}_{k}\left(f_{k}\right) / \gamma_{0 k}\left(b_{k}\right)\right) d f_{k}-r_{0} \\
& \left\{\log _{2}\left[\delta_{k} \dot{\gamma}_{k}\left(b_{k}\right)\right]-\frac{\partial}{\partial b_{k}}\left\{b_{k} \log _{2}\left[\gamma_{0 k}\left(b_{k}\right)\right]\right\}\right\}
\end{aligned}
$$




$$
\begin{aligned}
& \times\left(B_{k}-b_{k}-c_{k}\right) \\
& -\int_{0}^{b_{k}} \log _{2}\left[\delta_{k} \dot{\gamma}_{k}\left(f_{k}\right)\right] d f_{k} \\
& +\int_{0}^{b_{k}} \log _{2}\left[\gamma_{0 k}\left(b_{k}\right)\right] d f_{k}-r_{0} \\
= & \left\{\log _{2}\left[\delta_{k} \dot{\gamma}_{k}\left(b_{k}\right)\right]-\log _{2}\left[\gamma_{0 k}\left(b_{k}\right)\right]\right\} \\
& \times\left(B_{k}-b_{k}-c_{k}\right) \\
& -\left\{\frac{1}{\ln 2} \frac{b_{k}}{\gamma_{0 k}\left(b_{k}\right)} \frac{\partial}{\partial b_{k}}\left\{\gamma_{0 k}\left(b_{k}\right)\right\}\right\} \\
& \times\left(B_{k}-b_{k}-c_{k}\right)-\int_{0}^{b_{k}} \log _{2}\left[\delta_{k} \dot{\gamma}_{k}\left(f_{k}\right)\right] d f_{k} \\
& +b_{k} \log _{2}\left[\gamma_{0 k}\left(b_{k}\right)\right]-r_{0} .
\end{aligned}
$$

We can write the above expression in a simpler form:

$$
\frac{\partial}{\partial b_{k}} \dot{\xi}_{k}\left(b_{k}, c_{k}\right)=g\left(b_{k}, c_{k}\right)-r_{0},
$$

and we can substitute expressions (A.5)-(A.9) to formula (B.1) to obtain the derivative of function $\dot{\xi}_{k}\left(b_{k}, c_{k}\right)$ for the rural channel model and the expression for $g_{k}\left(b_{k}\right)$ :

$$
\begin{aligned}
g_{k}\left(b_{k}\right) \simeq & \left\{\log _{2}\left\{A_{k} \delta_{k}\left[1+\cos \left(2 \pi b_{k} \tau_{k}-\varphi_{k}\right)\right]\right\}\right\} \\
& \cdot\left(B_{k}-b_{k}-c_{k}\right) \\
& +\log _{2}\left\{b _ { k } ^ { - 1 } \left[\Gamma_{k}+\frac{1}{2 \pi \tau_{k} A_{k} \delta_{k}}\left[\operatorname{tg}\left(\pi b_{k} \tau_{k}-\frac{\varphi_{k}}{2}\right)\right.\right.\right. \\
& \left.\left.\left.\left.\times\left(B_{k}-b_{k}-c_{k}\right)+\frac{b_{k}}{\ln 2} \frac{\varphi_{k}}{2}\right)\right]\right]\right\} \\
& \times\left\{\Gamma_{k}+\frac{1}{2 \pi \tau_{k} A_{k} \delta_{k}}\left[\operatorname{tg}\left(\pi b_{k} \tau_{k}-\frac{\varphi_{k}}{2}\right)-\operatorname{tg}\left(\frac{\varphi_{k}}{2}\right)\right]\right\} \\
& \times\left\{2 A_{k} \delta_{k} \cos ^{2}\left(\pi b_{k} \tau_{k}-\frac{\varphi_{k}}{2}\right)\right\}^{-1}\left(B_{k}-b_{k}-c_{k}\right) \\
& -\int_{0}^{b_{k}} \log _{2}\left\{A_{k} \delta_{k}\left[1+\cos \left(2 \pi f_{k} \tau_{k}-\varphi_{k}\right)\right]\right\} d f_{k} \\
& -b_{k} \log _{2}\left\{\frac { 1 } { b _ { k } } \left[\Gamma_{k}+\frac{1}{2 \pi \tau_{k} A_{k} \delta_{k}}\left[\operatorname{tg}\left(\pi b_{k} \tau_{k}-\frac{\varphi_{k}}{2}\right)\right.\right.\right. \\
&
\end{aligned}
$$

\section{References}

[1] Z. Zhang, Y. He, and E. K. P. Chong, "Opportunistic scheduling for OFDM systems with fairness constraints," Eurasip
Journal on Wireless Communications and Networking, vol. 2008, Article ID 215939, 2008.

[2] Z. Han, Z. Ji, and K. J. R. Liu, "Fair multiuser channel allocation for OFDMA networks using Nash bargaining solutions and coalitions," IEEE Transactions on Communications, vol. 53, no. 8, pp. 1366-1376, 2005.

[3] C. Sacchi, F. Granelli, and C. Schlegel, "A QoE-oriented strategy for OFDMA radio resource allocation based on minMOS maximization," IEEE Communications Letters, vol. 15, no. 5, pp. 494-496, 2011.

[4] J. Chen and A. L. Swindlehurst, "Applying bargaining solutions to resource allocation in multiuser MIMO-OFDMA broadcast systems," IEEE Journal of Selected Topics in Signal Processing, vol. 6, no. 2, pp. 127-139, 2012.

[5] Z. Han, Z. Ji, and K. J. R. Liu, "Non-cooperative resource competition game by virtual referee in multi-cell OFDMA networks," IEEE Journal on Selected Areas in Communications, vol. 25, no. 6, pp. 1079-1090, 2007.

[6] S. Buzzi, G. Colavolpe, D. Saturnino, and A. Zappone, "Potential games for energy-efficient power control and subcarrier allocation in uplink multicell OFDMA systems," IEEE Journal of Selected Topics in Signal Processing, vol. 6, no. 2, pp. 89-103, 2012.

[7] D. Wu, D. Yu, and Y. Cai, "Subcarrier and power allocation in uplink OFDMA systems based on game theory," in Proceedings of the IEEE International Conference Neural Networks and Signal Processing (ICNNSP '08), pp. 522-526, June 2008.

[8] D. Yu, D. Wu, Y. Cai, and W. Zhong, "Power allocation based on power efficiency in uplink OFDMA systems: a game theoretic approach," in Proceedings of the 11th IEEE Singapore International Conference on Communication Systems (ICCS '08), pp. 92-97, November 2008.

[9] F. Chen, L. Xu, S. Mei, T. Zhenhui, and L. Huan, "OFDM bit and power allocation based on game theory," in Proceedings of the IEEE International Symposium on Microwave, Antenna, Propagation, and EMC Technologies for Wireless Communications (MAPE '07), pp. 1147-1150, August 2007.

[10] H. Yu, L. Gao, Z. Li, X. Wang, and E. Hossain, "Pricing for uplink power control in cognitive radio networks," IEEE Transactions on Vehicular Technology, vol. 59, no. 4, pp. 1769$1778,2010$.

[11] H. Kwon and B. G. Lee, "Distributed resource allocation through noncooperative game approach in multi-cell OFDMA systems," in Proceedings of the IEEE International Conference on Communications (ICC '06), pp. 4345-4350, July 2006.

[12] L. Wang, Y. Xue, and E. Schulz, "Resource allocation in multicell OFDM systems based on noncooperative game," in Proceedings of the IEEE 17th International Symposium on Personal, Indoor and Mobile Radio Communications (PIMRC '06), pp. 1-5, September 2006.

[13] Z. Liang, Y. H. Chew, and C. C. Ko, "Decentralized bit, subcarrier and power allocation with interference avoidance in multicell OFDMA systems using game theoretic approach," in Proceedings of the IEEE Military Communications Conference (MILCOM '08), pp. 1-7, November 2008.

[14] F. Wang, M. Krunz, and S. Cui, "Price-based spectrum management in cognitive radio networks," IEEE Journal on Selected Topics in Signal Processing, vol. 2, no. 1, pp. 74-87, 2008.

[15] S. K. Jayaweera, G. Vazquez-Vilar, and C. Mosquera, "Dynamic spectrum leasing: a new paradigm for spectrum sharing in cognitive radio networks," IEEE Transactions on Vehicular Technology, vol. 59, no. 5, pp. 2328-2339, 2010. 
[16] D. Niyato and E. Hossain, "Competitive pricing for spectrum sharing in cognitive radio networks: dynamic game, inefficiency of nash equilibrium, and collusion," IEEE Journal on Selected Areas in Communications, vol. 26, no. 1, pp. 192-202, 2008.

[17] H. Bogucka, "Efficient and rational spectrum utilization in opportunistic OFDMA networks with imperfect CSI: a utilitybased top-down approach," Wireless Communications and Mobile Computing, vol. 12, no. 5, pp. 431-444, 2012.

[18] G. Hardin, "The tragedy of the commons," Science, vol. 162, no. 3859, pp. 1243-1248, 1968.

[19] J. Perez-Romero, O. Sallent, R. Agusti, and L. Giupponi, "A novel on-demand cognitive pilot channel enabling dynamic spectrum allocation," in Proceedings of the 2nd IEEE International Symposium on New Frontiers in Dynamic Spectrum Access Networks (DySPAN '07), pp. 46-54, Dublin, Ireland, April 2007.

[20] G. J. Foschini and J. Salz, "Digital communications over fading radio channels," The Bell System Technical Journal, vol. 62, no. 2, pp. 429-456, 1983.

[21] S. M. Perlaza, M. Debbah, S. Lasaulce, and H. Bogucka, "On the benefits of bandwidth limiting in decentralized vector multiple access channels," in Proceedings of the 4th International Conference on Cognitive Radio Oriented Wireless Networks and Communications (CROWNCOM '09), Hannover, Germany, June 2009.

[22] P. Straffin, Game Theory and Strategy, The Mathematical Association of America, 2002. 

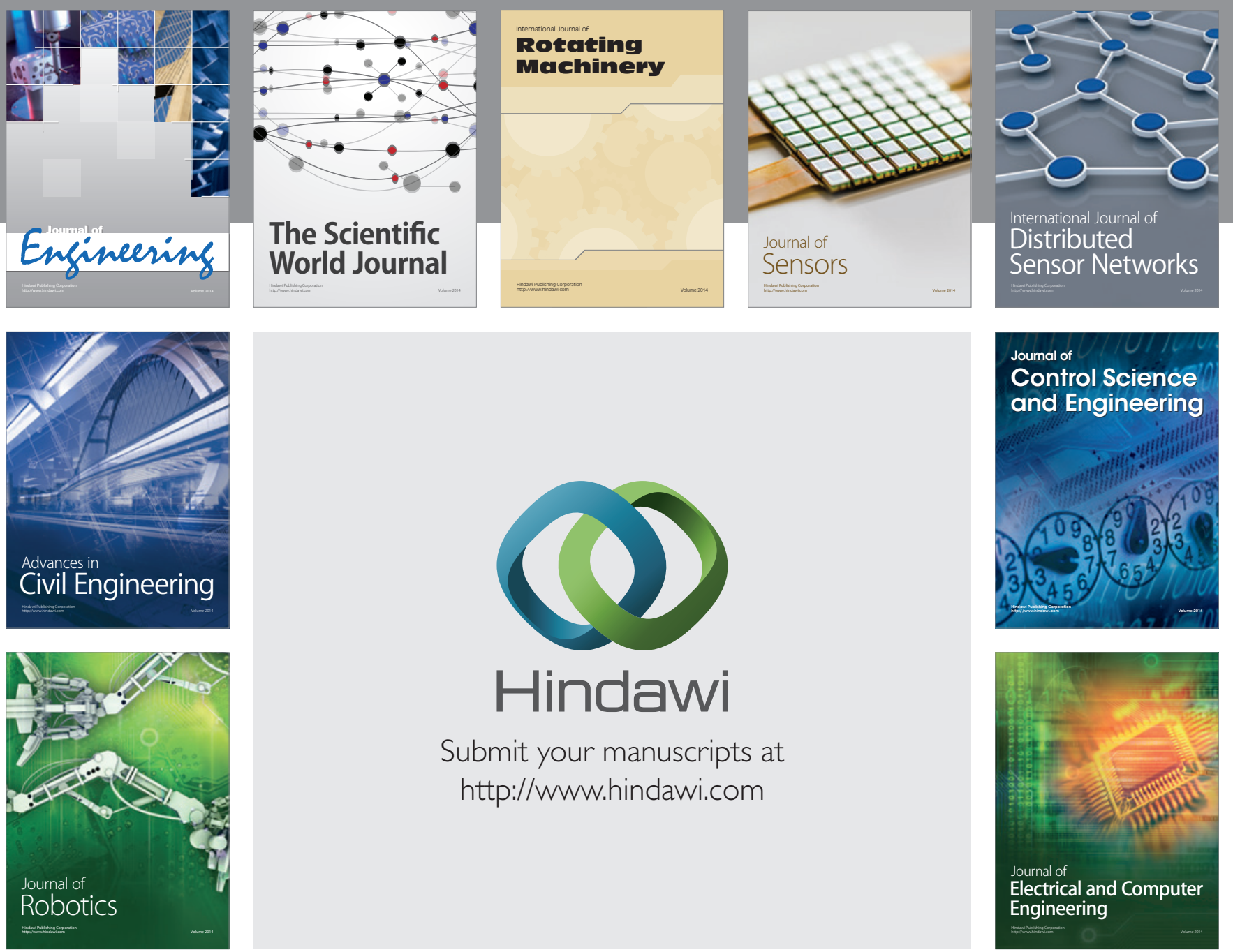

Submit your manuscripts at

http://www.hindawi.com
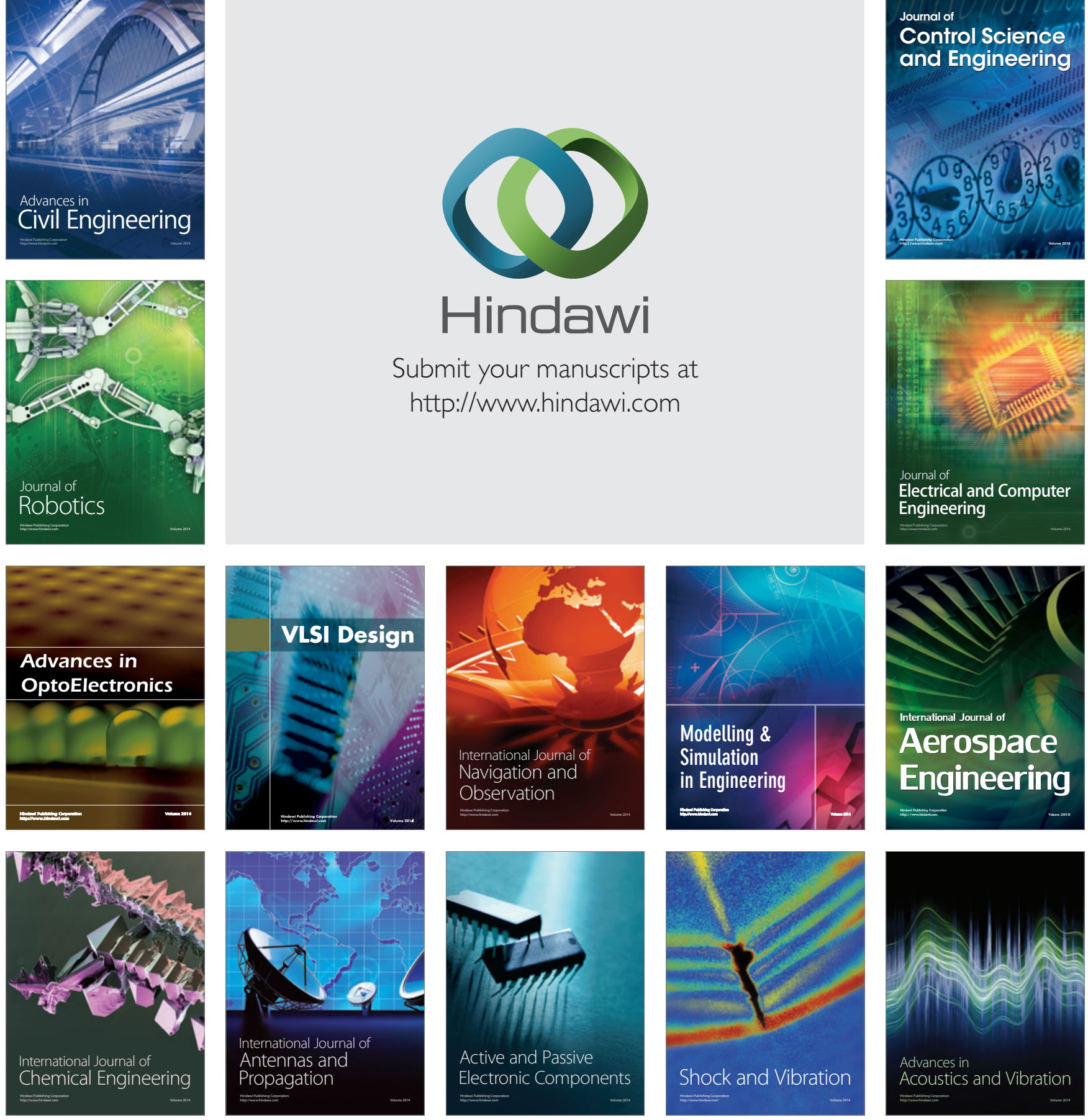